\title{
Editorial to selected papers from the 1st IMEKO TC19 Workshop on METROLOGY FOR THE SEA
}

\author{
Fabio Leccese \\ "Roma Tre" University of Rome, Science Department, via della Vasca Navale n.84, 00146 Roma, Italy
}

\begin{abstract}
Section: EDITORIAL
Citation: Fabio Leccese, Editorial to selected papers from the 1st IMEKO TC19 Workshop on METROLOGY FOR THE SEA, Acta IMEKO, vol. 7, no. 2, article 1, June 2018, identifier: IMEKO-ACTA-07 (2018)-02-01
\end{abstract}

Editor: Dušan Agrež, University of LUBLANA, Slovenia

Received June 4, 2018; In final form June 4, 2018; Published June 2018

Copyright: @ 2018 IMEKO. This is an open-access article distributed under the terms of the Creative Commons Attribution 3.0 License, which permits unrestricted use, distribution, and reproduction in any medium, provided the original author and source are credited

Corresponding author: Fabio Leccese, email: fabio.leccese@uniroma3.it

Dear Reader,

the Sea has been the element that more than other represented for humanity the sense of unknown with its intrinsic luggage of fears and doubts, maybe representing the first barrier that humanity had to overcome in an organized way, thus representing the first challenge towards Knowledge.

Because its complexity, the Sea is studied from several points of view involving many research fields. The first edition of IMEKO TC19 Workshop on METROLOGY FOR THE SEA held in the prestigious and renowned "Parthenope" University of Naples on 11th-12th October 2017, represented a first meeting point between scientists coming from many scientific fields which given their vision of subjects dear to the Measures both theoretical and practical. In fact, the concept of measurement uncertainty is faced in the particular operative context (high pressures, operative logistic problems, etc.) that, in some cases, stresses the instrumentation, which present technical characteristics extremely pushed to face the problems typical of the sea.

In this perspective, the Congress won the challenge to reach a first general objective to promote the scientific debate and the encounter of different sensibilities around the typical topics of the "measurements worlds" promoting a better and deeper comprehension and harmonization of them.

The first Edition of the Congress gathered more than 100 papers by researchers of all over the world, whose almost 50 accepted and published. Among this relevant number of contributions, the board of Congress selected a few papers to be extended and submitted for the peer review of ACTA IMEKO journal. A first selection of the papers eligible for the Special Issue has been done from the Congress board, then, the successive fundamental contribute of the journal reviewers to which my special thanks go, determined eight papers considered worthy of publication in this journal and which represent a synthesis of the scientific activity in this field.

In the first paper, E. Petritoli and F. Leccese examine the development of an attitude and control system for a tailless Autonomous Underwater Vehicle without movable control surfaces. For it, it is extremely interesting the AUV manoeuvring system managed acting, with high accuracy, on the buoyancy system and on the centre of gravity displacement, which control is realized by an ad hoc control system called Glider Integrated Control System. The device has a wide wing surface allows a smooth glide slope and an excellent operational range.

In order to highlight their strengths and weaknesses, E. Romano et al. deal a critical review of the main methods used for sampling, measuring and classifying marine sediments whose knowledge is useful in many research areas as sediment contamination, ecology of benthic communities, seismic studies, remote sensing surveys and beach nourishment. This revision shows as the highest accuracy of grain size data is obtained using flexible strategies and adopting suitable sampling devices and analytical instruments based on the characteristics of the study area and the specific aim of the survey.

In order to improve absolute positioning provided in GNSS context, S. Gaglione et al. propose to jointly use well-known quality indicators like the signal-to-noise ratio and the satellite elevation angle with an additional indicator, the redundancy number. The benefits of using the proposed weighting schemes is demonstrated in urban canyon and with single (GPS only) or multiple (GPS/Glonass) constellations. In fact, the position errors, using the weighting scheme based on redundancy numbers, are significantly reduced, for all the considered configurations. 
F. Arrichiello et al. present an overview of the recent and ongoing research activities of the Italian Interuniversity Centre on Integrated Systems for the Marine Environment (ISME) in the field of geophysical seismic survey. Such activities, performed in the framework of the H2020 European project WiMUST, include the development of technologies and algorithms for Autonomous Surface Crafts and Autonomous Underwater Vehicle to perform geophysical seismic survey by mean of a team of robots towing streamers equipped with acoustic sensors.

S. Buogo et al. present the EMPIR "UNAC-LOW" project, which aims at developing the European metrological capacity for the calibration of hydrophones and autonomous noise recording systems for frequencies below $1 \mathrm{kHz}$. The project will allow improving the EU metrological capacities for absolute measurement of underwater sound, with a direct effect on the implementation of regulation and EU Directives that require underwater acoustic measurements to be traceable. A coherent EU metrology strategy for underwater acoustics will be developed as one of the main project outcomes.

In the sixth paper, R. Marsili et al. show an interesting example of a new and fast solution for 3D modelling, based on the same principles of photogrammetry: the Photo-modelling. The comparison between this and the metrological technique of structured light 3D scanning, provided by the Creaform Go Scan 50 with metrological certification, is the aim of this paper, defining performances and verifying the potential of this innovative, simple and economical technique. In order to investigate the alignment error due to a poor geometry reconstruction many test have been performed. The results show different performances, in terms of deviation distributions and volumetric errors.
G. D'Onghia et al. show some practical applications of the MEMO (Marine Environment MOnitoring system) lander, with indications of relative advantages and limitations. MEMO is a baited lander equipped with 2 video cameras, a multi-parametric probe and a current meter. MEMO is able to explore and monitor marine ecosystems in the Mediterranean Sea for 24 consecutive hours survey even down to $1000 \mathrm{~m}$ in depth. During its activity, data on environmental parameters (depth, salinity, temperature, current) and related to bentho-pelagic species (small-scale distribution, size and behaviour), have been recorded.

G. Gaglianone et al. show the application of a particular geophysical method: the "GeoDive", which allows surveying with high accuracy nearshore shallow waters with a low budget. The authors tested and tuned the "GeoDive" method allowed to map the submerged morphologies and to acquire highresolution optical images for further photogrammetric processing. By coupling the processing of underwater-acquired data obtained by a high definition Action Sport Cam, with the direct surveys performed by underwater SCUBA operators, it was possible to perform some morphological and sedimentological measurements and observations on the experimental targets and obtain 3D high-resolution models even in low visibility conditions.

The fishermen know that the sea is dangerous and the storm terrible, but they have never found these dangers sufficient reason for remaining ashore. (Vincent Van Gogh).

Fabio Leccese

Guest Editor 